\title{
Significant COVID-19 burden in Polish children
}

\author{
Teresa Jackowska ${ }^{1}$, August Wrotek ${ }^{1}$, Mateusz Jankowski², Jarosław Pinkas
}

${ }^{1}$ Department of Paediatrics, Medical Centre of Postgraduate Education, Warsaw, Poland ${ }^{2}$ School of Public Health, Medical Centre of Postgraduate Education, Warsaw, Poland

Submitted: 2 July 2020

Accepted: 23 July 2020

Arch Med Sci

DOI: https://doi.org/10.5114/aoms.2020.98407

Copyright $\odot 2020$ Termedia \& Banach

\begin{abstract}
Introduction: COVID-19 cases have rarely been reported in children. We sought to analyse the attack rate in paediatric population in Poland, focusing on local variations among the provinces, correlation with the number of tests per capita, and test positivity rate.

Material and methods: This cross-sectional study involved the 38.38 million population and detected 17,921 cases (age known in 17,822). Data were collected from publicly available registries and were analysed by age group and province of the country.

Results: Children constituted $6.68 \%$ of cases $(n=1,191)$. The attack rate reached $15.49 / 100,000$ children, increasing with age $(10.79 / 100,000$ in $<4$ y.o. to $21.59 / 100,000$ in 15-19 y.o.). Significant variations in the attack rates were observed: a 9.52-fold ratio between the highest and the lowest attack rates in provinces. The provinces from the first and fourth attack rate quartiles differed in the test positivity rate $(4.96 \%$ vs. $1.98 \%, p<0.05)$, but not in the number of tests per capita. The lowest quartile provinces showed 1.87- to 5.78-fold lower attack rates, compared to the directly neighbouring provinces, without any known population susceptibility differences. The attack rates in children and adults correlated very strongly (rho $=0.81)$. The attack rate correlated with the test positivity rate ( $r h o=0.64$ in children and rho $=0.71$ in adults) but not with the number of tests per capita.

Conclusions: COVID-19 burden in children is significant. The local differences highlight various testing strategies, but the awareness of SARS-CoV-2 in children is essential. The correlation between attack rates in children and adults shows that the outbreak in children is parallel to the one observed in adults.
\end{abstract}

Key words: children, COVID-19, attack rates.

\section{Introduction}

The first confirmed case of the coronavirus disease (COVID-19) caused by severe acute respiratory syndrome coronavirus 2 (SARS-CoV-2) was reported in December 2019 in China, whereas the first case in Poland was diagnosed on $4^{\text {th }}$ March 2020 [1]. Globally, the pandemic has already caused over 5.5 million cases and 350 thousand deaths (as of $27^{\text {th }}$ May 2020) [2]. COVID-19 cases have mainly been reported in adults, while the paediatric population is believed to remain relatively free of the SARSCoV-2 infection and safe, because the disease is associated with a milder course and a positive outcome [3]. The proportion of children requiring hospitalisation varies. In Italy, it has been assessed to be marginal (5.2\% of sick children in Italy, as of $10^{\text {th }}$ April 2020) [3], while in Spain more than

\author{
Corresponding author: \\ Prof. Teresa Jackowska \\ Department of Paediatrics \\ Medical Centre \\ of Postgraduate Education \\ 99-103 Marymoncka St \\ 01-813 Warsaw, Poland \\ E-mail: tjackowska@cmkp. \\ edu.pl
}


one fourth of all diagnosed paediatric cases were inpatients (26\% as of $29^{\text {th }}$ May 2020) [4]. Nevertheless, the varying hospitalisation rates may result both from the divergent treatment standards and the testing strategies. The need for intensive care also varies between $1.2 \%$ [3] and 3.7\% [4]. The estimates from the USA, which is the most heavily affected country, report between $5.7 \%$ and $20 \%$ of children needing hospital treatment, and $0.58-2 \%$ requiring intensive care unit treatment [5]. In general, the paediatric quota to the total burden of COVID-19 is low, accounting for $1 \%$ to $5 \%$ of all diagnosed cases, but the frequency differs substantially among the various countries [6]. Although reports on deaths in children are scarce [5], paediatricians still need to remain vigilant. Recently, an alert has been raised due to the in crease in the incidence of a Kawasaki-like disease related to multisystem inflammatory syndrome in children (MIS-C) [7-9]. Moreover, children could play a role in the spread of the disease, and paediatric transmission might even be crucial to the reopening after the lockdown [10]. Data on the attack rate in children is lacking because the great majority of efforts, especially in countries heavily affected by COVID-19, is directed towards the diagnosis and treatment of those in the most severe condition; thus, the attack rate in the paediatric population may be underestimated.

We sought to analyse the number of cases and the attack rate in different age groups in Poland, to verify the local variations between the provinces and the correlation with the number of tests, number of tests per 1000 inhabitants, and the test positivity rate.

\section{Material and methods}

\section{Definitions}

Only laboratory-confirmed cases of the SARSCoV-2 infection were taken into consideration; nasopharyngeal swabs were collected; the real-time polymerase chain reaction (RT-PCR) was used as a reference method; and one positive result was found to be relevant, irrespective of the clinical presentation, because the signs and symptoms, especially in paediatric patients, are diverse, and asymptomatic courses have also been reported [3, $11,12]$. The data are collected on a daily basis and derive from the Chief Sanitary Inspectorate, which is the public administration authority responsible for epidemiological surveillance, including the outbreak of the novel coronavirus. Testing strategy: testing was available at public healthcare facilities (including hospitals, ambulances dedicated to swabbing administered by the local healthcare facilities and sanitary inspectorates, and drivethrough points) and performed free of charge upon a clinical or epidemiological premise.

\section{Study setting}

This is a cross-sectional study, involving the whole population of Poland, and all the SARSCoV-2 cases from the first case ( $4^{\text {th }}$ March) until $15^{\text {th }}$ May 2020 were eligible for the study. According to the statistics of Poland, the population of Poland amounted to 38,382,576 people, including 7,690,007 children. The demographic data are publicly available and are updated every 6 months; the most recent available dataset is valid for $31^{\text {st }}$ December 2019, and this was used as a reference for the computation of the attack rate [13]. The number of inhabitants in the database is presented in the following age groups: 0-4 years, 5-9 years, 10-14 years, and 15-19 years; hence, the age limit of a paediatric patient needed to be set at 19 years. Only rough estimates of the number of infants were used (data on the 2019 birth cohort has not been published yet); it was calculated as a difference between the total number of children in the age group 0-4 years and the number of live births in the preceding years. The demographic data for Poland and for each of the 16 administrative provinces in Poland have been considered, with no analysis of migration among the regions because no important variations in this field have been notified, and the demographic situation in Poland is rather constant. Thus, no significant bias should be attributable in these terms to the demographic data.

The endpoints of this study included the number of cases and the attack rate (per 100 thousand children) in the previously mentioned age groups (0-4 years, 5-9 years, 10-14 years, and 15-19 years). Respective values for adults were also obtained. In order to verify the attack rate homogeneity, the attack rate ratio for each age group (including adults) was computed as a quotient between the regions with the highest and the lowest attack rate. The number of tests per 100,000 inhabitants and the test positivity rate were calculated separately for each region.

All the procedures involving human subjects are in accordance with the 1964 Helsinki Declaration and its later amendments. Patients in epidemiological reports are anonymised, without the possibility of individual identification of any of the participants.

\section{Statistical analysis}

Then, provinces from the first and the last quartile with respect to the attack rate were compared in terms of the crude number of tests, the number of tests per 1000 inhabitants, and the test positivity rate. The Mann-Whitney $U$ test was used, and the values were presented as median and an interquartile range (IQR). 
Each of the provinces from the first and the last attack rate quartile was opposed against its neighbouring provinces; attack rates were compared (total, total in children, and in five paediatric age groups, creating a set of 28 comparisons). The number of tests per 1000 inhabitants and the test positivity rate were also compared.

Spearman's rank correlation test was executed in order to verify a correlation between the reported number of cases in the age groups and the attack rates, the number of tests, the number of tests per 1000 inhabitants, and the test positivity rate (separately for the regions). The results were expressed as the Spearman's rank correlation coefficient (rho). The level of significance was set at $p<0.05$. The statistical analysis was performed with Statistica 13.1 (StatSoft, Tulsa, OK, USA).

\section{Results}

As of $15^{\text {th }}$ May 2020, 17,921 cases of COVID-19 have been diagnosed in Poland, wherein precise data on the patient's age was known in 17,822 $(99.45 \%)$ cases. The paediatric contribution to the total number of cases was $6.68 \%(n=1191)$.

The highest number of cases was observed in children aged $15-19$ years ( $n=389$ cases, $32.7 \%)$, followed by $10-14$ ( $n=328,27.5 \%), 5-9(n=267$, $22.4 \%)$, and $<4$ years ( $n=207,17.4 \%$ ) (Table I). Interestingly, only 38 cases (3.2\% of cases among children) have been diagnosed in infants.

The total paediatric attack rate reached $15.49 / 100,000$, with the highest value observed in the oldest age group (15-19 years) - 21.59/100,000, decreasing then with a younger age $(16.24 / 100,000$ for 10-14-year-olds, $13.7 / 100,000$ for 5-9-year-olds, 10.79/100,000 for < 4 years of age); in infants it reached 10.12/100,000 (Table I).

According to the regions, the highest number of paediatric cases was diagnosed in the Silesian
Province ( $n=241,20.2 \%$ ), followed by Mazovian ( $n=149,12.5 \%)$, Lower Silesian, and Małopolskie ( $n=135, n=11.3 \%$ each) (Table II), but these are four of the five provinces with the highest number of children in Poland. The highest attack rate was observed in the Silesian Province (28.25/100,000), followed by Łódzkie $(25.11 / 100,000)$ and Lower Silesian (24.67/100,000). Interestingly, the geographical variation in the attack rates was significant, and the attack rate ratio (between the highest and the lowest attack rate regions) reached 9.52 for children in general, varying between 5.16 in those aged 0-4 years to 23 in 5-9-year-olds; in infants and in 10-14-year-olds it was not computable, because no cases were reported in at least one province. The attack rate ratio was even higher in adults, reaching 12.07 (Table I).

The total number of tests reached 491,186, with a mean of 12.8 tests per 1000 inhabitants, varying between $4.8 / 1000$ in the Opolskie Province to $26.6 / 1,000$ in the Mazovian Province, which happen to be the regions with the lowest and highest number of inhabitants, respectively. The test positivity rate varied between $0.98 \%$ (Warmian-Mazurian) and $11.62 \%$ (Silesian) with a mean of $3.63 \%$ (Table II).

We opposed the provinces from the first and fourth quartiles in terms of the attack rate in children, and found that they differed in test positivity rate $(4.96 \%$ vs. $1.98 \%, p<0.05)$ but not in the number of tests per 1000 inhabitants (11.11 vs. 8.78, $p=0.69$ ) (Table III). Furthermore, we opposed each of the provinces from the first and fourth quartile against directly neighbouring provinces. We found that in each of the first quartile regions the attack rates were higher than in their neighbours in 24 out of 28 comparisons (total attack rate, children in total, infants, 0-4, 5-9, 10-14, and 15-19 years old) with the exceptions of 5-9-year-olds in tódzkie, the total attack rate in tódzkie and Małopolskie, and the infants in Małopolskie. The

Table I. Case distribution, attack rates, and attack rate ratio in various age groups

\begin{tabular}{|lccccc|}
\hline Age group [years] & $\begin{array}{c}\text { Number of cases } \\
(\%)\end{array}$ & $\begin{array}{c}\text { Attack rate } \\
\text { [per 100,000] }\end{array}$ & $\begin{array}{c}\text { Lowest attack rate } \\
\text { [per 100,000] }\end{array}$ & $\begin{array}{c}\text { Highest attack rate } \\
\text { [per 100,000] }\end{array}$ & $\begin{array}{c}\text { Attack rate } \\
\text { ratio }\end{array}$ \\
\hline$<1$ & $38(3.2)$ & 10.12 & 0 & 32.32 & NA \\
\hline $0-4$ & $207(17.4)$ & 10.79 & 3.79 & 19.59 & 5.16 \\
\hline $5-9$ & $267(22.4)$ & 13.69 & 1.23 & 28.37 & 22.99 \\
\hline $10-14$ & $328(27.5)$ & 16.24 & 0 & 28.69 & NA \\
\hline $15-19$ & $389(32.7)$ & 21.59 & 5.2 & 42.71 & 8.21 \\
\hline Total (children) & $1191(6.68)$ & 15.49 & 2.97 & 28.25 & 9.52 \\
\hline Adults & $16,631(93.32)$ & 54.19 & 10.66 & 128.28 & 12.07 \\
\hline $\begin{array}{l}\text { Total (children } \\
\text { and adults) }\end{array}$ & $17,822(100)$ & 46.43 & 9.09 & 109.39 & 12.03 \\
NA - non-applicable. & & & & &
\end{tabular}




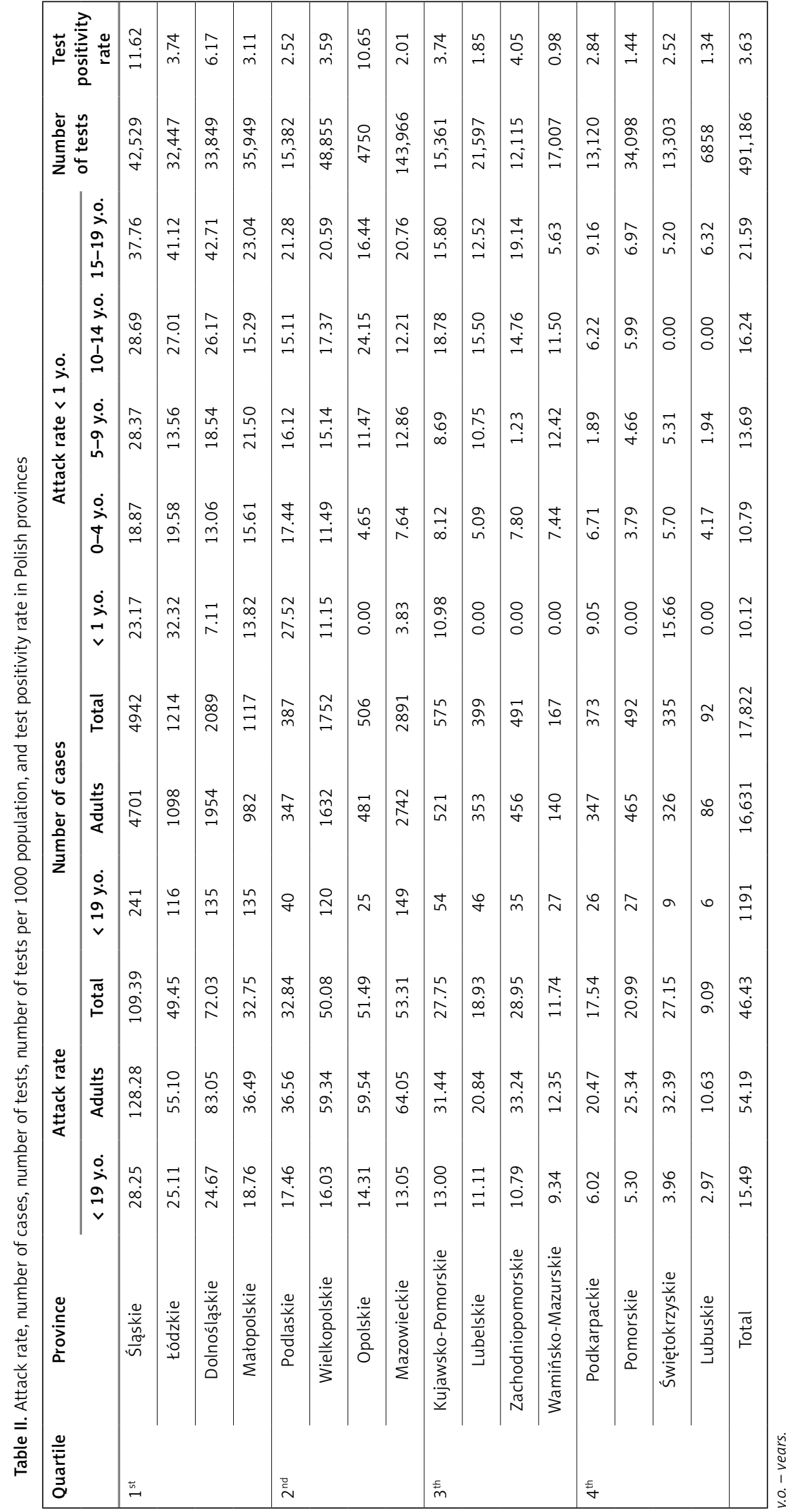


Table III. Comparison between the provinces from the $1^{\text {st }}$ and the $4^{\text {th }}$ quartile regarding the attack rate. The attack rates are expressed per 100,000 inhabitants

\begin{tabular}{|c|c|c|c|c|c|c|c|}
\hline \multirow[t]{2}{*}{ Variable } & \multicolumn{3}{|c|}{$1^{\text {st }}$ quartile provinces } & \multicolumn{3}{|c|}{$4^{\text {th }}$ quartile provinces } & \multirow[t]{2}{*}{$P$-value } \\
\hline & Median & LQ & UQ & Median & LQ & UQ & \\
\hline Attack rate < 19 y.o. & 24.89 & 21.71 & 26.68 & 4.63 & 3.46 & 5.66 & $<0.05$ \\
\hline Attack rate $<1$ y.o. & 18.50 & 10.46 & 27.75 & 4.52 & 0.00 & 12.36 & 0.2 \\
\hline Attack rate $0-4$ y.o. & 17.24 & 14.34 & 19.23 & 4.94 & 3.98 & 6.21 & $<0.05$ \\
\hline Attack rate 5-9 y.o. & 20.02 & 16.05 & 24.94 & 3.30 & 1.92 & 4.99 & $<0.05$ \\
\hline Attack rate $10-14$ y.o. & 26.59 & 20.73 & 27.85 & 2.99 & 0.00 & 6.10 & $<0.05$ \\
\hline Attack rate $15-19$ y.o. & 39.44 & 30.40 & 41.92 & 6.64 & 5.76 & 8.06 & $<0.05$ \\
\hline Attack rate adults & 69.07 & 45.79 & 105.66 & 22.91 & 15.55 & 28.87 & $<0.05$ \\
\hline Attack rate (total) & 60.74 & 41.10 & 90.71 & 19.26 & 13.31 & 24.07 & $<0.05$ \\
\hline Number of tests & 34,899 & 33,148 & 39,239 & 13,212 & 9989 & 23,701 & 0.11 \\
\hline Number of tests per 1000 & 11.11 & 9.98 & 12.44 & 8.78 & 6.47 & 12.66 & 0.69 \\
\hline Test positivity rate (\%) & 4.96 & 3.42 & 8.90 & 1.98 & 1.39 & 2.68 & $<0.05$ \\
\hline
\end{tabular}

$L Q$ - lower quartile, UQ - upper quartile, $p$-Mann-Whitney U test.

number of tests per 1000 inhabitants was higher in each of the regions (vs. neighbours) except for the Silesian Province. The test positivity rates were higher in Lower Silesian and Silesian Province $(6.2 \%$ vs. $5.2 \%$, and $11.6 \%$ vs. $5 \%$, respectively), but lower in Małopolskie and Łódzkie Province (3.1\% vs. $5.7 \%$, and $3.7 \%$ vs. $5.7 \%$, respectively) (Table IV).

To the contrary, the provinces from the last quartile had significantly lower attack rates than neighbours in 27 out of 28 comparisons (except for attack rate in infants in Świętokrzyskie Province), and the number of tests per 1000 was generally lower (but higher in Pomorskie Province as compared to its neighbours). The attack rate in children was 1.87 - to 5.78 -fold lower than in the neighbouring provinces, suggesting an underestimation rate in the fourth quartile regions. Unexpectedly, the test positivity rate was lower than in the neighbours (with an exception of Podkarpackie, which showed a slightly higher rate than its neighbours: $2.8 \%$ vs. $2.5 \%$, repsectively) (Table IV).

Spearman's rank correlation test revealed a very strong correlation between the attack rate in children and the attack rate in adults (rho = 0.81 ), and the number of cases did correlate with the attack rates (rho $=0.80$ in children, rho $=$ 0.85 in adults). A correlation between the attack rates among adults and children decreased with decreasing age (rho $=0.81$ in 15-19-year-olds, through rho $=0.7$, to rho $=0.59$ in those aged $0-4$ years). A strong correlation between the test positivity rate and attack rate (rho $=0.64$ in children and rho $=0.71$ in adults) and between the attack rate in children (not in adults) and the number of tests in the provinces (rho $=0.52$ ) was observed. Notably, there was no correlation between the attack rate and the number of tests per capita (or per 1000 inhabitants in the region) (Table V). As of $15^{\text {th }}$ May 2020, two deaths due to the COVID-19 were reported among people aged 18 years, with no deaths in younger patients.

\section{Discussion}

This study reveals a surprisingly high percentage of COVID-19 cases attributable to children (6.68\%), which translates into a significant attack rate $(15.49 / 100,000)$ in the paediatric population in Poland. At first, paediatric cases were reported rarely, but with the spread of the outbreak, the number of infected children has increased [11]. The interim estimates in the US showed that children accounted for up to $5 \%$ of all cases [14], but the most recent data reported reduced the percentages of paediatric illness: as of $2^{\text {nd }}$ April 2020 $1.7 \%$ of 149,082 cases in the US were diagnosed in children (i.e. those under 18 years) [5]. These numbers are in line with those observed during the epidemic peak in China, when paediatric cases corresponded to approximately $2 \%$ of diagnosed patients [11]. The European data show a low percentage of patients in the paediatric population: $1.4 \%$ in Italy and $1.5 \%$ in the UK $[3,15]$, while reports from Spain show even lower numbers: in the group of children up to 14 years old (the next age group is set broadly as 15-29 years old) the percentage totalled $0.6 \%$ (out of 248,068 cases, as of $29^{\text {th }}$ May 2020) [4]. On the other hand, the most recent data from Canada [16] report a much 


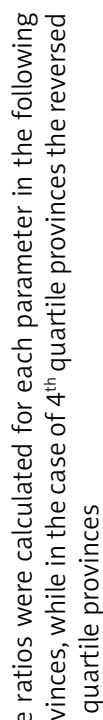

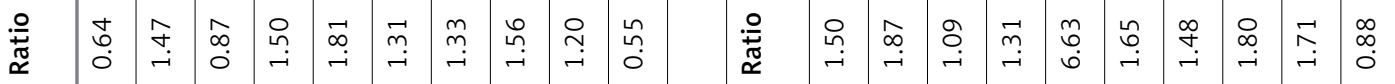

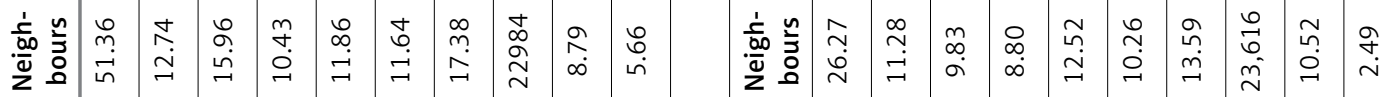

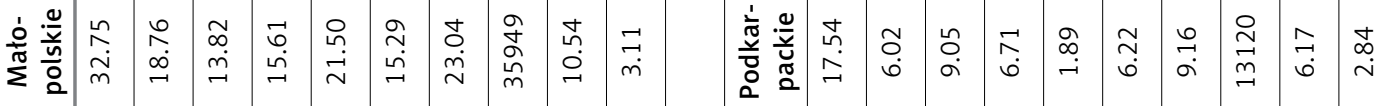

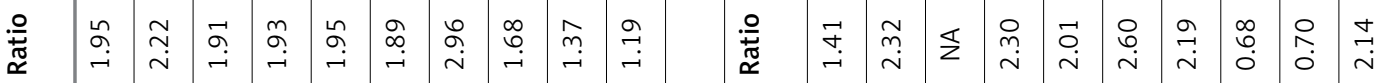

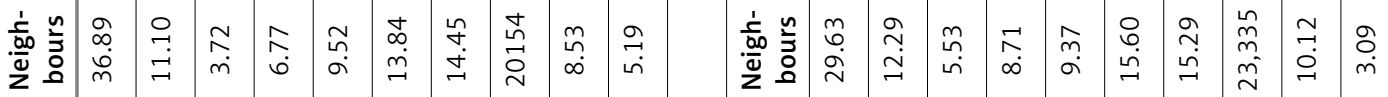

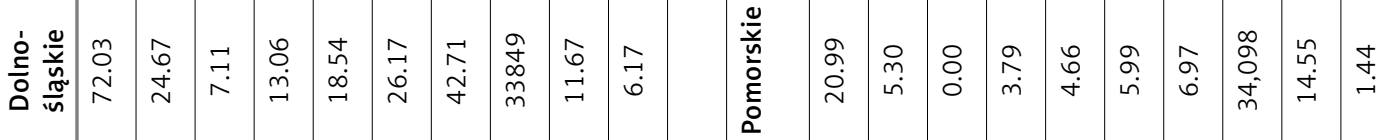

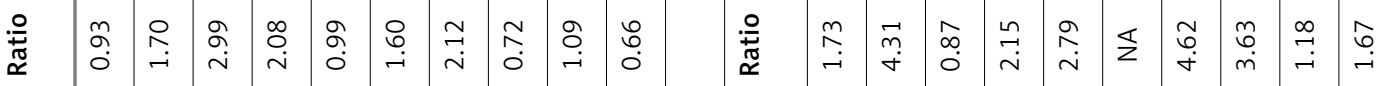

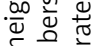

至

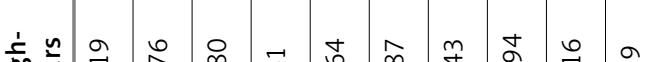

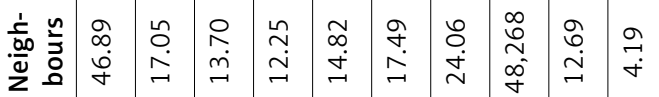

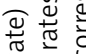

完

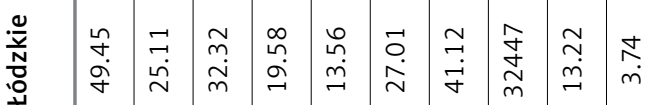

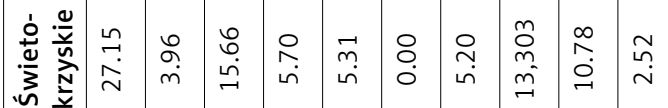

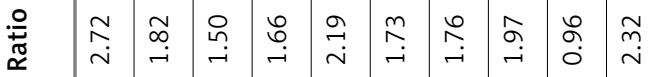

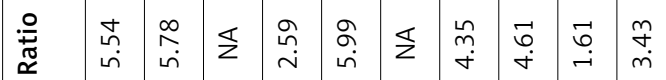

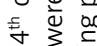

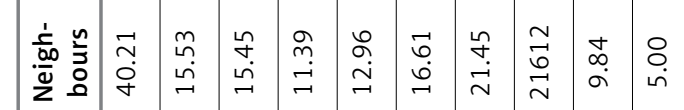

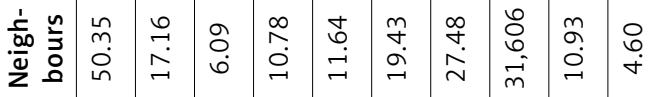

它

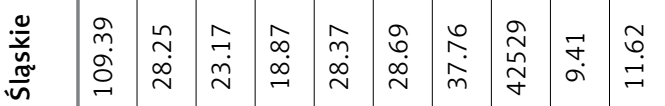

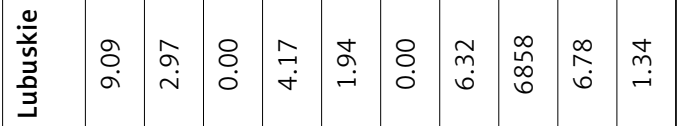

जी है जी

耘

ठ욤

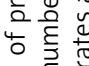

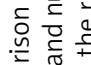

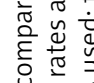

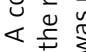

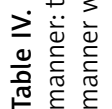

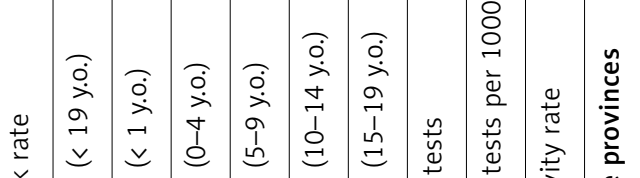

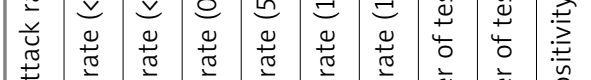

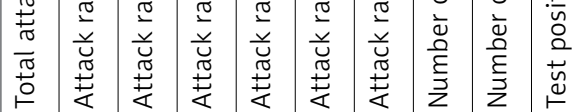

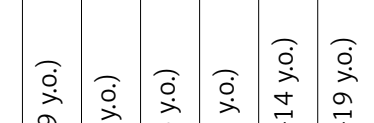

8

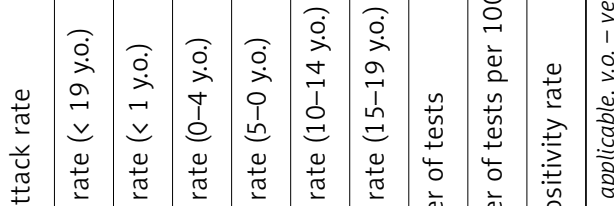

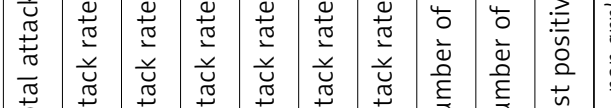


higher percentage $(5.66 \%)$ of COVID-19 cases in children ( 2306 out of 40,746 cases) in comparison with other countries, and our data (with a slightly higher proportion of $6.68 \%$ ) is in line with the Canadian results.

This high percentage of cases found in children may arise from higher (relative or absolute) detection of COVID-19 in children (or in a particular population, e.g. Polish or Canadian children) or lower detection in adults, but the attack rate is a more independent indicator, and shows intriguing values. The attack rate in children in Poland (as of $15^{\text {th }}$ May 2020) is between those reported in the general population in Algeria and Argentina at the time of writing (14.96 and 15.9 per 100,000, respectively), meaning that the paediatric population may and should also be considered affected [17]. Interestingly, the current attack rate in Argentina has increased 2.4 -fold (31 $1^{\text {st }}$ May vs. $15^{\text {th }}$ May 2020). Differences among the countries may result from different testing strategies. Moreover, a certain degree of underestimation needs to be considered because we observe huge variations between the provinces in Poland, which did not derive from an extremely different local epidemiological situation. The influence of the approach to testing is highlighted by considerable differences between the extreme attack rates in Polish provinces (almost 10-fold difference between the highest and the lowest), although it could result from varying epidemic situations and differences in the settings of the SARS-CoV-2 transmission (e.g. epidemic outbreaks in healthcare facilities or nursing homes) among the provinces. An analysis of four provinces with the lowest attack rate showed more than five-times lower attack rates than in their direct neighbours. We emphasise that the provinces in Poland refer to the administrative divisions only, without any further known inequality regarding population susceptibility, virus exposition, or health-care accessibility. There may be minor divergences in the social behaviour patterns, which could have influenced the attack rate in Polish children, but there are no data to explain such a huge regional discrepancy. Of note, the attack rate correlated with the total number of tests, but not with the number of tests per 1000 population. Hence, any efforts to establish a cut-off value of the number of tests per population that would be sufficient to guarantee a true reflection of the attack rate are not reasonable at this moment. In general, the number of diagnosed cases increases with the number of tests, but the lack of the correlation with the test-per-population rate probably means that this issue is reflected only partially, and to a different degree in various locations.

A correlation between the attack rate and the test positivity rate underlines the role of the test-

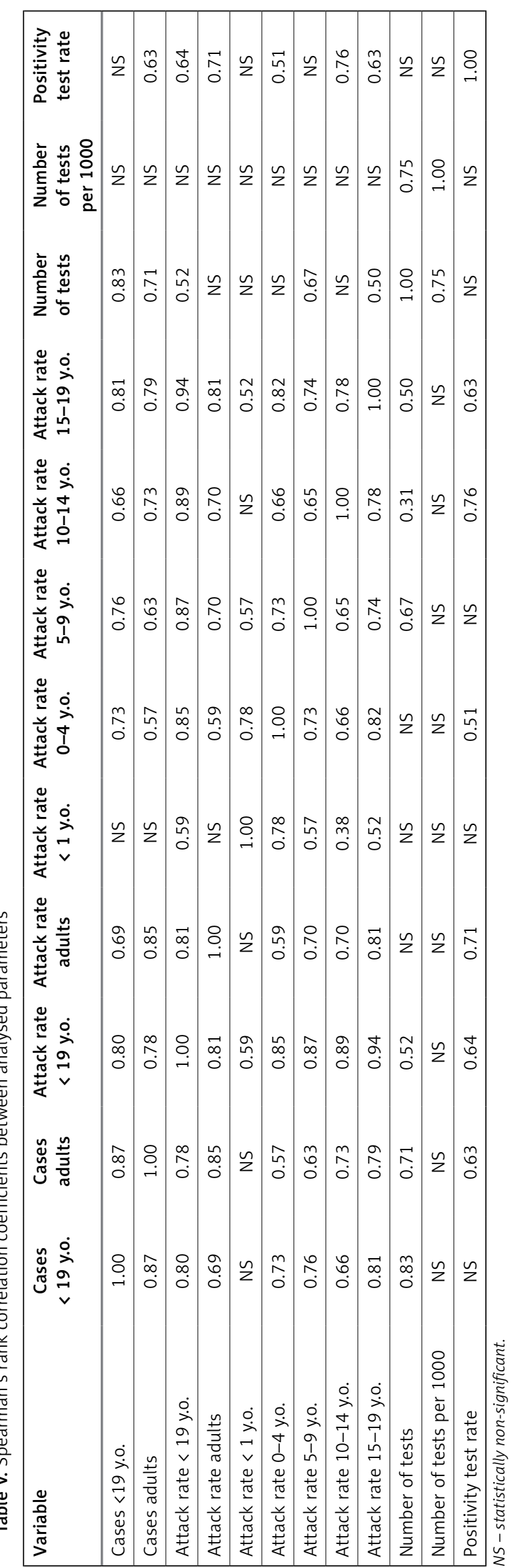


ing strategies. Optimally, if everyone is tested, a higher attack rate is seen in a higher test positivity rate, thus reflecting a genuinely higher incidence. Nonetheless, the differences between the neighbouring regions contradict this time-point in testing, although the test positivity rate in Poland is low. A comparison of the situation between Poland and Canada explains the differences: many of the characteristics between these two countries are similar (population: ca. 38.4 vs. 37.8 million), including the percentage of paediatric cases, but there are differences in the number of tests and, first of all, in the attack rate (roughly fourfold higher in Canada) [17]. If we analysed only the number of tests per capita (the mean number of tests per inhabitant is 2.5 -fold lower in Poland; $12.8 / 1000$ in Poland versus 31.2/1000 in Canada, as of $12^{\text {th }}$ May 2020) [18], we could falsely argue that the number of cases in Poland is severely underestimated. However, the positivity test rate in Poland is 1.6-fold lower than in Canada (3.7\% vs. $6 \%$ ), which suggests that the attack rate and the true number of cases in Canada is simply higher [17]. Thus, the number of tests should be analysed in close relation with the test positivity rate.

If Poland was at another time-point of the epidemic, the percentage of cases diagnosed in children might decrease; this might also be the case for the US, taking into account a decrease from $5 \%$ to $1.7 \%$ within less than a month [5, 14]. An asymptomatic, mild, or moderate disease course, which has been reported in $97 \%$ of laboratory-confirmed cases in China [12], clearly carries the risk of an underdiagnosis. In the case of a huge outbreak and rapidly shrinking medical resources, the diagnostic efforts may be focused on patients in a more severe health condition, thus decreasing the number of tests in children in favour of adults. It is crucial to ensure stable access to SARS-CoV-2 testing in the paediatric population because it mirrors the attack rate in adults. Our study showed a strong correlation between the attack rate in children and adults ( $r$ ho $=0.81$ ), which increased with the children's age. This in turn suggests that the epidemic attacks both groups, but in paediatric patients it is seen to a lower degree. We can hypothesise that the number of potential sources of SARS-CoV-2 infection is higher among adults (especially those occupationally active) than in children, and settings of SARS-CoV-2 transmission may differ between age groups. Poland did not face a depletion of the health care system resources directly, and the functioning of the paediatric hospital care (where the majority of tests have been performed) did not deteriorate, which may explain the relatively high participation of paediatric patients in the total number of cases. Additionally, alongside with the long incubation period, the missed paediatric cases increase the children's transmission capacity [11]. The question on the possible role of children in the spread of the disease needs to be raised. To date, the majority of studies argue against the significant role of children in the spread of the disease [19]. A Swiss study by Posfay-Barbe traced 39 household contacts and revealed that a child presented symptoms prior to adult contact only in $8 \%$ of cases, being a possible index case [20]. In a Chinese study by $\mathrm{Wu}$, only marginal transmission from children was observed: $96 \%$ of children analysed in a series of 69 paediatric patients had previously been in contact with adult index cases [21], while another Chinese study reported one in 10 children to transmit the SARS-CoV-2 to adult contacts [22]. Interestingly, if children played an important role in the spread of the SARS-CoV-2, then an in creased number of paediatric cases could precede the outbreak in adults, and time-series analyses should answer this question.

Notably, the percentage of cases in the age groups in our study reflects the study by the CDC COVID-19 Response Team, who found the highest proportion in 15-17-year-olds, followed by $10-14$-year-olds ( $32 \%$ and $27 \%$, respectively) [5]. Conversely, taking into account only laboratory-confirmed cases, a lower percentage was observed in the oldest group of patients by Dong et al. $(21.5 \%)$ in favour of $6-10$-year-olds $(23.4 \%$, which is similar to our result of $22.4 \%$ in $5-9$-yearolds) [12]. Both studies found $26-30 \%$ of cases in the youngest group of children; $26 \%$ in those aged under four years old by the CDC COVID-19 Response Team, and 30.5\% under five years old by Dong et al., while our investigation shows a lower contribution of this age group (17.4\% under four years of age), mainly due to the distinctly low number of cases in infants (3.2\% only). These results are even more with contrasting the proportion of cases in infants reported by Garazzino et al., who revealed $39.3 \%$ (out of a group of 168 children) in children under one year of age [3]. The authors speculate, however, that this result might originate from a social tendency to seek a paediatric consultation more frequently, alongside the paediatricians' inclination towards hospital admission of infants [3].

Previous analysis of paediatric populations showed that children are frequently asymptomatic carriers of viruses or bacteria [23]. Due to the lack of specific symptoms and asymptomatic course of COVID-19, the testing strategy for SARSCoV-2 should consider testing children living in one household with a quarantined person [24]. As of $15^{\text {th }}$ May, Poland does not report deaths due to COVID-19 in people under 18 years. The risk of dying from COVID-19 is highest in the oldest age 
groups, and knowledge about the pathogenesis of severe manifestations of COVID-19 is constantly increasing [25]. To protect high-risk populations, extensive social isolation rules were introduced in many countries, including Poland. The COVID-19 pandemic has resulted in social isolation and "national quarantine" that has forced changes in social behaviour. A significant group of elderly people have isolated from their relatives and often avoid contact with their grandchildren, which is supposed to limit transmission from children to grandparents [26]. The sociological and psychological consequences of social isolation should be considered when planning strategies to counteract the second wave of SARS-CoV-2 infections in Europe [26].

There exist certain strengths and weaknesses of this study. The enrolment of each diagnosed paediatric COVID-19 case in the whole population of a middle-sized European country (approximately 38 million citizens) constructed a huge sample of 1191 paediatric patients. Apart from the size of the group, a strict rule of including only laboratory-confirmed cases is another advantage, which decreases the risk related to misclassification. However, the true number of cases is certainly higher, and the burden could be assessed more precisely by a comparison of the number of tests performed in children. Unfortunately, this type of data was not available when preparing this paper. Secondly, due to the retrospective character of the analysis, data on preventive measures used by children and their parents/tutors is lacking, and there are no data on direct/indirect contact with the sources; nevertheless, it was not within the scope of the aim of the study. Also, the age definition used in this study may be confusing because we set the age limit at 19 years old due to the reasons explained above. In general, the definition of a paediatric population varies among countries, thus complicating the comparisons with other studies, also in terms of reports on COVID-19.

In conclusion, the most important finding of this study is that the COVID-19 burden in children is significant. Both the number of cases and the attack rate in children are not marginal and should not be neglected. We might be underestimating the paediatric cases, becasue they do not require rapid emergency actions, and the cases are less "spectacular" than in adults. The awareness of the possibility of COVID-19 affecting children needs to be maintained. There is a correlation between the attack rates in children and adults, and it increases with the children's age, showing that the outbreak in children is parallel to the one observed in adults. The role of a judicious testing strategy is crucial for the recognition of the COVID-19 burden in children, and pharmacoeconomic analyses of various testing strategies are necessary.

\section{Acknowledgments}

We are grateful to all the physicians, nurses, and paramedics involved directly in the diagnostic process, and the treatment of patients suspected of COVID-19. We would also like to express our sincere gratitude to the employees of the State Sanitary Inspection for collecting and sharing data.

The study was supported by the CMKP grant number 501-1-020-19-20

\section{Conflict of interest}

The authors declare no conflict of interest.

\section{References}

1. Gujski M, Raciborski F, Jankowski M, Nowicka PM, Rakocy K, Pinkas J. Epidemiological analysis of the first 1389 cases of COVID-19 in Poland: a preliminary report. Med Sci Monit 2020; 26: e924702.

2. https://coronavirus.jhu.edu/map.html, accessed on $27^{\text {th }}$ May 2020.

3. Garazzino S, Montagnani C, Donà D, et al. Multicentre Italian study of SARS-CoV-2 infection in children and adolescents, preliminary data as at 10 April 2020. Euro Surveill 2020; 25: 2000600

4. Análisis de los casos de COVID-19 notificados a la RENAVE hasta el 10 de mayo en España. https://www.isciii.es/ QueHacemos/Servicios/VigilanciaSaludPublicaRENAVE/ EnfermedadesTransmisibles/Documents/INFORMES/ Informes\%20COVID-19/Informe\%20n\%2033.\%20Análisis\%20de\%20los\%20casos\%20de\%20COVID-19\%20 hasta $\% 20$ el\%2010\%20de\%20mayo\%20en\%20España\%20a\%2029\%20de\%20mayo\%20de\%202020.pdf

5. CDC COVID-19 Response Team. Coronavirus Disease 2019 in Children - United States, February 12-April 2, 2020. MMWR Morb Mortal Wkly Rep. 2020; 69: 422-6.

6. Ludvigsson JF. Systematic review of COVID-19 in children shows milder cases and a better prognosis than adults. Acta Paediatr 2020; 109: 1088-95.

7. Riphagen S, Gomez X, Gonzalez-Martinez C, Wilkinson N, Theocharis P. Hyperinflammatory shock in children during COVID-19 pandemic. Lancet 2020; 395: 1607-8.

8. Chiotos K, Bassiri H, Behrens EM, et al. Multisystem Inflammatory Syndrome in Children during the COVID-19 pandemic: a case series. J Pediatric Infect Dis Soc 2020; 9: 393-8.

9. Verdoni L, Mazza A, Gervasoni A, et al. An outbreak of severe Kawasaki-like disease at the Italian epicentre of the SARS-CoV-2 epidemic: an observational cohort study. Lancet 2020; 10.1016/S0140-6736(20)31103-X. doi:10.1016/S0140-6736(20)31103-X.

10. Viner RM, Whittaker E. Kawasaki-like disease: emerging complication during the COVID-19 pandemic. Lancet 2020; 10.1016/S0140-6736(20)31129-6. doi:10.1016/ S0140-6736(20)31129-6.

11. She J, Liu L, Liu W. COVID-19 epidemic: disease characteristics in children. J Med Virol 2020; 10.1002/ jmv.25807. doi:10.1002/jmv.25807.

12. Dong Y, Mo X, Hu Y, et al. Epidemiology of COVID-19 among children in China. Pediatrics 2020; 145: e20200702.

13. http://demografia.stat.gov.pl/bazademografia/CustomSelectData.aspx?s=lud\&y=2019\&t=00, accessed on 25th May 2020. 
14. CDC COVID-19 Response Team. Severe Outcomes Among Patients with Coronavirus Disease 2019 (COVID-19) - United States, February 12-March 16, 2020. MMWR Morb Mortal Wkly Rep 2020; 69: 343-6.

15. Docherty $A B$, Harrison EM, Green $C A$, et al. Features of 20133 UK patients in hospital with covid-19 using the ISARIC WHO Clinical Characterisation Protocol: prospective observational cohort study. BMJ 2020; 369: m1985.

16. https://health-infobase.canada.ca/covid-19/epidemiological-summary-covid-19-cases.html\#a3, accessed on $24^{\text {th }}$ May 2020 , at 11 am EDT.

17. https://qap.ecdc.europa.eu/public/extensions/ COVID-19/COVID-19.html, accessed on 31 $1^{\text {st }}$ May 2020.

18. https://art-bd.shinyapps.io/covid19canada/, accessed on $12^{\text {th }}$ May 2020.

19. Lee B, Raszka WV Jr. COVID-19 transmission and children: the child is not to blame. Pediatrics 2020; e2020004879. doi:10.1542/peds.2020-004879.

20. Posfay-Barbe KM, Wagner N, Gauthey M, et al. COVID-19 in children and the dynamics of infection in families. Pediatrics 2020; 146: e20201576.

21. Wu Q, Xing Y, Shi L, et al. Co-infection and other clinical characteristics of COVID-19 in children. Pediatrics 2020; 146: e20200961.

22. Cai J, Xu J, Lin D, et al. A case series of children with 2019 novel coronavirus infection: clinical and epidemiological features. Clin Infect Dis 2020; ciaa198. doi:10.1093/cid/ ciaa198.

23. Będzichowska A, Przekora J, Stapińska-Syniec A, et al. Frequency of infections caused by ESBL-producing bacteria in pediatric ward - single center five-year observation. Arch Med Sci 2019; 15: 688-93.

24. Sierpiński R, Pinkas J, Jankowski M, et al. Sex differences in the frequency of gastrointestinal symptoms and olfactory or taste disorders in 1942 nonhospitalized patients with coronavirus disease 2019 (COVID-19). Pol Arch Intern Med 2020; 130: 501-5.

25. Bhaskar S, Sinha A, Banach M, et al. Cytokine Storm in COVID-19 - Immunopathological Mechanisms, Clinical Considerations, and Therapeutic Approaches: The REPROGRAM Consortium Position Paper. Front Immunol 2020; 11: 1648.

26. Burlacu A, Mavrichi I, Crisan-Dabija R, et al. "Celebrating old age": an obsolete expression during the COVID-19 pandemic? Medical, social, psychological, and religious consequences of home isolation and loneliness among the elderly. Arch Med Sci 2020. doi:10.5114/ aoms.2020.95955. 